2016 - Volume: 17 Number: 3

Page: 585 - 593

DOI : $10.18038 / \mathrm{btda} .47955$

Received: 21 June 2016

Revised: 08 September 2016

Accepted: 21 September 2016

\title{
THE EFFECT OF TRIANGULAR CAVITIES ON PIEZOELECTRIC PROPERTY OF GRAPHENE
}

\author{
M. Menderes ALYÖRÜK* \\ Computer Education and Instructional Technologies Dept., Faculty of Education, \\ Dumlupınar University, Kütahya, TURKEY
}

\begin{abstract}
Piezoelectricity is a unique material property that converts mechanical energy to electrical one or vice versa. In order to call a matter piezoelectric, it should be in non-centrosymmetric structure and have sufficiently large band gap. Graphene has none of these properties in its natural composition. It is shown that, coaxing the graphene structure can give piezoelectric property to this non-piezoelectric material. In this study, the size effect of the triangular holes and their placements on the structure to the piezoelectricity are investigated theoretically via density functional theory based calculations. According to the calculation results, while the size of the cavity effects the piezoelectricity, layout of the similar shaped triangular cavities do not change the piezoelectric coefficient.
\end{abstract}

Keywords: Graphene, piezoelectricity, DFT, 2D structures

\section{INTRODUCTION}

Piezoelectricity is a unique property of the semiconductors or insulators with non-centrosymmetric structures. Piezoelectricity is the ability of certain materials to generate an electric charge in response to mechanical stress or vice versa. From the discovery of the piezoelectric effect [1] to present day, researchers endeavor to find natural or artificial effective piezoelectric materials to meet the needs of time. Most commonly used piezoelectric materials are in bulk phases and they are not suitable to produce nano-dimensional devices. Besides, vast majority of effective three dimensional piezoelectric materials, such as the most commonly used $\mathrm{Pb}(\mathrm{Zr}, \mathrm{Ti}) \mathrm{O}_{3}$ or $\mathrm{PZT}$-based compositions, contain more than $60 \%$ mass of toxic lead.

Today's technological demands compel researchers to find novel, piezoelectric materials to construct nano-sized devices. Reproducibility of single layered structures from their bulk phases was shown with the isolation of the single layer graphene from the multi layered graphite. This development encourages the researchers to investigate electronic, optical and photonical properties of these materials and bring up to their availability in next generation nanophotonic and nanoelectronic applications [2-4]. And also, monolayer materials show different properties when compared to their multilayer structures [5]. For example, two dimensional structures no longer protect their inversion symmetry which they have in bulk phases [6]. This situation enables these two dimensional materials to exhibit piezoelectric properties which they do not have in their bulk structures. Experimental observation of the graphene and other two-dimensional materials [7-9] has paved the way of production of ultra-thin structures with innovative physical properties. The target of this technology is to keep under control the nanoscale deformation and motion. In order to provide the dynamical control of the graphene based non-piezoelectric structures under applied electric field, it is necessary to acquire piezoelectric properties.

For the last few years researchers trying to find the answer of how graphene can exhibit piezoelectric property. To achieve that, first centrosymmetry must be corrupt and then its needed to investigate if the forbidden energy gap is formed or not. Jin et.al [10] reported that the formation of the pretty neat

*Corresponding Author: menderes.alyoruk@dpu.edu.tr 
triangular holes during the experimental growth of the hexagonal boron nitride structures by the secondary effect of the electron beam. With the high-resolution transmission electron microscopy (HRTEM) images they showed the holes can be formed in different sizes but in the same directions (Figure 1). Sharma and Chandratreise [11], reported that graphene can show piezoelectric property by coaxing it with nonsymmetrical holes. They showed that the triangular hole breaks centrosymmetry and transforms the graphene into piezoelectric. Their results are close to natural piezoelectric materials, quartz $(\sim \% 72)$ and boron nitride $(\sim \% 36)$. Reed and Ong [12] reported that by adding extra atoms ( $\mathrm{Li}$, $\mathrm{K}, \mathrm{H}, \mathrm{F}$ and their combinations $\mathrm{H}$ and $\mathrm{F}, \mathrm{F}$ and $\mathrm{Li}$ ) to the graphene structure it is possible to make graphene piezoelectric. Calculated piezoelectric coefficients differentiates related with the add-atom, and the highest piezoelectric coefficient is $5.5 \times 10^{-11} \mathrm{Cm}^{-1}[12]$ when compared to 2D graphene like boron nitride structure $3.71 \times 10^{-10} \mathrm{Cm}^{-1}$ [13]. $\mathrm{d}_{31}$ strain piezoelectric coefficient of the graphene oxide structures were calculated as $0.24 \mathrm{pmV}^{-1}$ by Chang et. al [14]. This value is comparable with the maximum $\mathrm{d}_{31}$ coefficient of the engineered piezoelectricity of graphene $0.3 \mathrm{pmV}^{-1}$ [12], and three dimensional piezoelectrics such as wurtzite $\mathrm{GaN}$ and boron nitride (BN); $0.96 \mathrm{pmV}^{-1}$ [15] and $0.33 \mathrm{pmV}^{-1}$ [16], respectively. Band-piezoelectric effect on biaxial strained graphene was studied by Wang et. al. According to their results, application of biaxial strain enables the formation of a small $(\sim 90 \mathrm{meV})$ bandgap on graphene and this brings $37 \mathrm{nCN}^{-1}$ piezoelectric coefficient which is twice as high compared to traditional piezoelectric materials [17]. Rodrigues et. al experimentally measured the piezoelectric coefficient of the single-layer graphene deposited on the $\mathrm{Si} / \mathrm{SiO}_{2}$ substrates as $1.4 \mathrm{nmV}^{-1}$, this value is twice of the modified lead zirconate titanate [18]. Technological requirements, wide range of application area and availability of reproducibility of high quality samples and also theoretical and experimental proof of the piezoelectric response of these two dimensional structures, opened the way of using them as pressure sensors, transducers, high voltage generators, etc [19-21]. Beside all these advantages, this novel two dimensional materials have the capability to take the place of lead contained piezoelectrics.

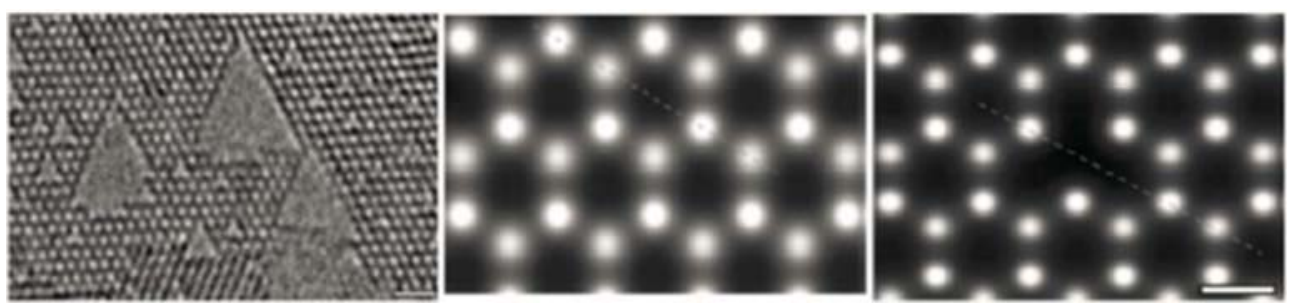

Figure 1. HRTEM images of the holes on h-BN structure[10]

In this work, density functional theory (DFT) based theoretical calculations were held to investigate the placement and size effect of the triangular holes on the piezoelectric performance of the graphene.

\section{MATERIALS AND METHODS}

Within the scope of current study, first-principles calculations based on the density functional theory, as implemented in Vienna Ab-Initio Simulation Package (VASP) [22, 23], are performed. Generalized gradient approximation (GGA) with in the Perdew-Burke-Ernzerhof (PBE) Formulation [24] and the Heyd-Scuseria-Ernzerhof (HSE06) hybrid functionals [25, 26] are used to treat the exchange-correlation interactions. The single electron wave functions are expanded into plane waves with the $600 \mathrm{eV}$ kinetic energy cut-off. 1x8x8 k-point mesh is used for structure optimizations with in the Monkhorst pack scheme [27]. $10^{-7}$ and $10^{-3} \mathrm{eV} / \mathrm{A}$ convergence criterion set for the electronic and ionic optimizations, respectively. In order to minimize the interactions between the layers $\sim 12 \mathrm{~A}$ vacuum space is set. In order to apply the strain in a desired direction properly hexagonal structure is transformed into $2 \mathrm{H}$ mode, which is composed of two hexagonal cells [13]. Berry's phase approximation [28] is used to investigate the polarization change under applied uniform strain, ranging from $0.02 /-0.02$ in steps of 0.01 , along the 
$y$ direction of the rectangular cell. Piezoelectric tensor, $\mathrm{e}_{\mathrm{ij}}$ is related with the $\varepsilon_{\mathrm{j}}$, polarization change along the $\mathrm{j}$ direction as mentioned below $[23,29]$,

$$
\mathrm{e}_{\mathrm{ij}}=\frac{\partial \mathrm{P}_{\mathrm{i}}}{\partial \varepsilon_{\mathrm{j}}}=\left(\frac{\partial \mathrm{P}_{\mathrm{i}}}{\partial \varepsilon_{\mathrm{j}}}\right)_{\mathrm{u}}+\sum_{\mathrm{k}}\left(\frac{\partial \mathrm{P}_{\mathrm{i}}}{\partial \mathrm{u}_{\mathrm{ik}}}\right)\left(\frac{\partial \mathrm{Pu}_{\mathrm{ik}}}{\partial \varepsilon_{\mathrm{j}}}\right)
$$

here, $\mathrm{P}_{\mathrm{i}}$ denotes the polarization change along the direction $i$, as a result of the $\varepsilon_{\mathrm{j}}$, strain applied in the $j$ direction. The first term in Equation (1) is named as the clamped-ion and defines the contribution of the homogeneous strain to the piezoelectric tensor. Second term comes from the internal relaxation of the ions. $\mathrm{u}_{\mathrm{ik}}$ denotes the fractional coordinate of the $i$ th ion along the $\mathrm{k}$ direction. Because of the crystal symmetry, the number of the independent piezoelectric tensor coefficients is reduced. For the graphene, only the $\mathrm{e}_{11}$ component of the piezoelectric stress tensor is needed. $\mathrm{e}_{11}$ relates the in plane strain to inplane electrical polarization. For each applied strain ions are kept in strained positions and clamped-ion piezoelectric properties are computed.

\section{RESULTS}

As mentioned above, triangular holes can be formed during the experimental growth of two-dimensional structures [10] and from the theoretical results of the Sharma and Chandratreise [11], formation of triangular holes can convert non-piezoelectric graphene to piezoelectric. From this point of view, to clarify the size effect of the holes, two structure are prepared and named as $12 \mathrm{H}$ and $18 \mathrm{H}$, Figure 2-a and Figure 3-a, related with the number of the $\mathrm{H}$ atoms, which is used for passivation of the edges of triangular holes. Bandstructure and piezoelectric coefficients are calculated using the relaxed structure parameters which was obtained after optimization procedures. Lattice parameters and bond lengths were given in Table 1. for the structures under study. Calculation results showed that, coaxing graphene with triangular holes can induce forbidden energy gaps with the values $0.46 \mathrm{eV}$ and $0.69 \mathrm{eV}$ for $12 \mathrm{H}$ and $18 \mathrm{H}$ structures, respectively. Now, structures with triangular holes, break the centrosymmetry of the graphene and energy gap formed. Applying the Berry's phase approximation to the $12 \mathrm{H}$ and $18 \mathrm{H}$ structures, polarization change according to applied uniform strain calculated and given in Figure 4. From Equation 1 the slope of the lines in Figure 4 gives the piezoelectric coefficients of the structures under study, $1.704 \times 10^{-10} \mathrm{C} / \mathrm{m}^{2}$ and $0.359 \times 10^{-10} \mathrm{C} / \mathrm{m}^{2}$, respectively.

Table 1. Cell parameters, bond lengths and the distances between triangular holes obtained from the optimization calculations. In units of Angstrom

\begin{tabular}{|c|c|c|c|c|c|c|}
\hline \multirow[t]{2}{*}{ Structure } & \multicolumn{3}{|c|}{ Cell Parameters } & \multicolumn{2}{|c|}{ Bond Lengths } & \multirow{2}{*}{$\begin{array}{c}\text { Distance } \\
\text { Between } \\
\text { Holes }\end{array}$} \\
\hline & a & b & c & $\mathrm{C}-\mathrm{C}$ & C-H & \\
\hline Graphene & 12.185 & 25.718 & 22.272 & 1.425 & - & -- \\
\hline Figure 2-a & 12.185 & 25.718 & 22.272 & 1.424 & 1.083 & -- \\
\hline Figure 3-a & 12.218 & 25.676 & 22.472 & 1.435 & 1.090 & -- \\
\hline Figure 5-a & 12.185 & 25.718 & 44.544 & 1.426 & 1.086 & 12.336 \\
\hline Figure 6-a & 12.185 & 25.718 & 44.544 & 1.428 & 1.095 & 14.642 \\
\hline Figure 7 -a & 12.185 & 25.718 & 44.544 & 1.424 & 1.083 & 14.615 \\
\hline
\end{tabular}




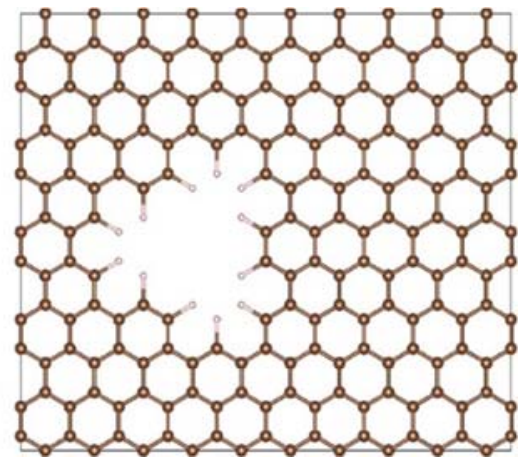

(a)

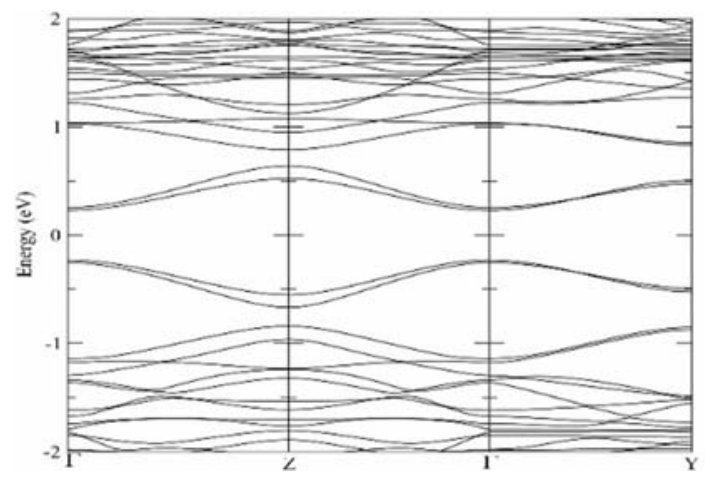

(b)

Figure 2. a) Structure image; brown atoms denote $\mathrm{C}$ and pink atoms denote $\mathrm{H}$ b) band structure graphic of $12 \mathrm{H}$ graphene

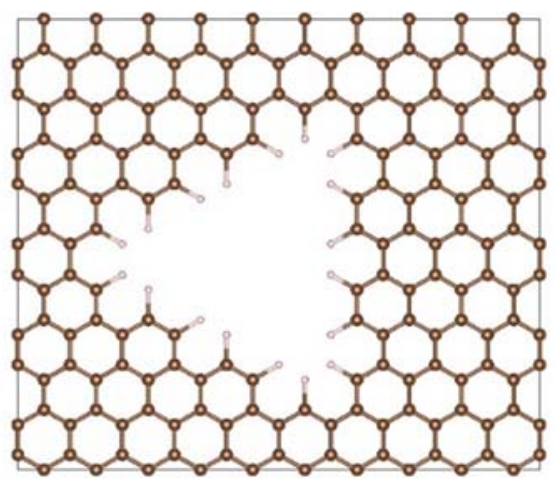

(a)

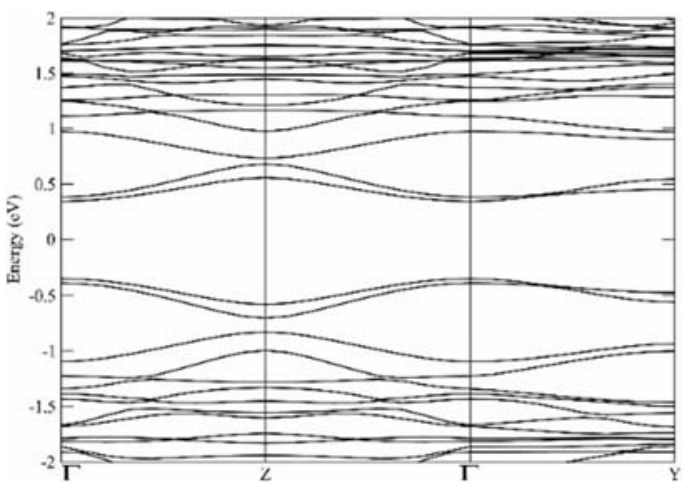

(b)

Figure 3. a) Structure image; brown atoms denote $\mathrm{C}$ and pink atoms denote $\mathrm{H}$ b) band structure graphic of $18 \mathrm{H}$ graphene

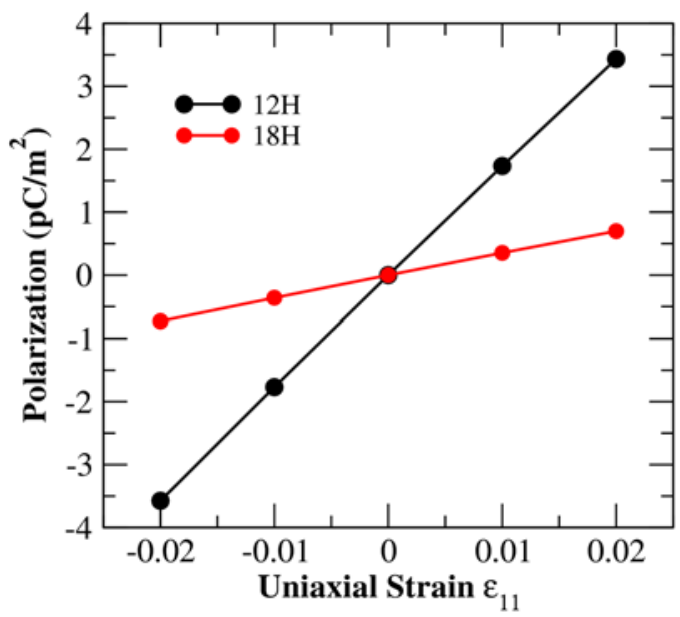

Figure 4. Polarization change under applied uniform strain for different sized triangular holes on graphene 
In order to see the effect of the triangular hole placements on piezoelectricity of the graphene, $12 \mathrm{H}$ structure is enlarged to three different alignments. These sequences are named according to the orientation of the pointed end of the triangles as face-same, face-in and face-out which were shown in Figure 5-a, 6-a, 7-a. In order to prevent the interaction between triangular holes $\sim 14$ Angstrom distance set. All of the structures optimized according to above procedure, cell parameters and atom positions were used to calculate band structures and piezoelectric coefficients. Calculation results reveal that the forbidden band gap values differs related with the layouts of the triangular holes. Calculated energy gap values for the structures given in Figure 5-6-7 are $0.1 \mathrm{eV}, 0.31 \mathrm{eV}$ and $0.63 \mathrm{eV}$, respectively. Differently from the base structure( $12 \mathrm{H}$ structure), forbidden energy gaps occur at $\mathrm{Z}$ point of the Brillouin zone for the face-in and face-out structures.

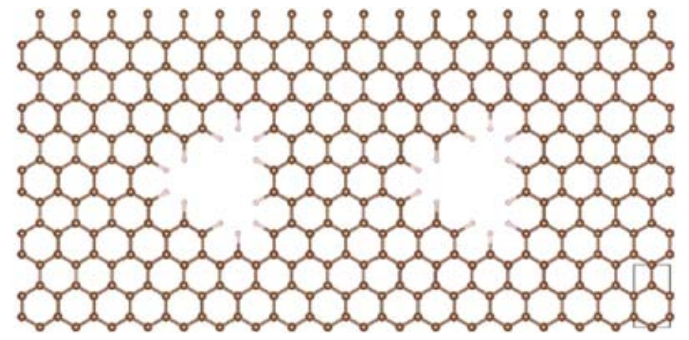

(a)

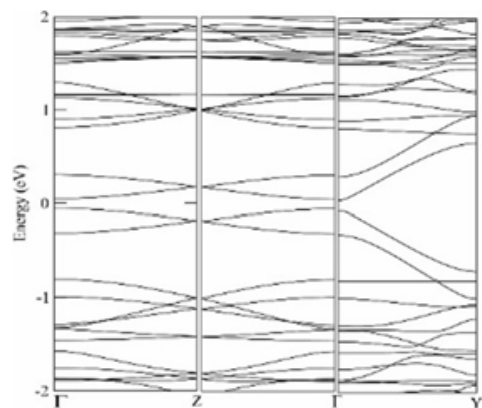

(b)

Figure 5. a) Placements of the holes; brown atoms denote $\mathrm{C}$ and pink atoms denote $\mathrm{H}$ b) bandstructure of the facesame structure

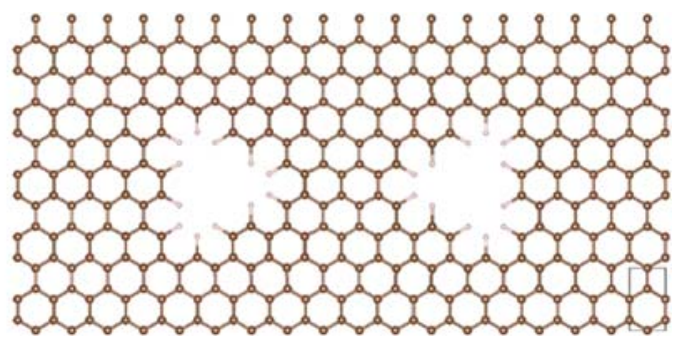

(a)

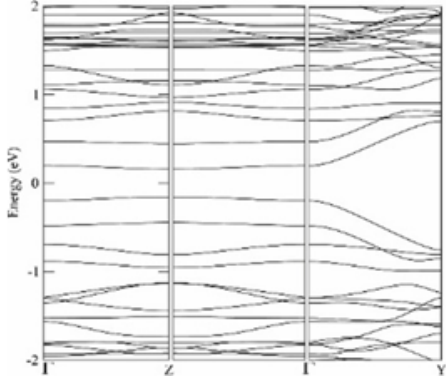

(b)

Figure 6. a) Placements of the holes; brown atoms denote $\mathrm{C}$ and pink atoms denote $\mathrm{H}$ b) bandstructure of the facein structure

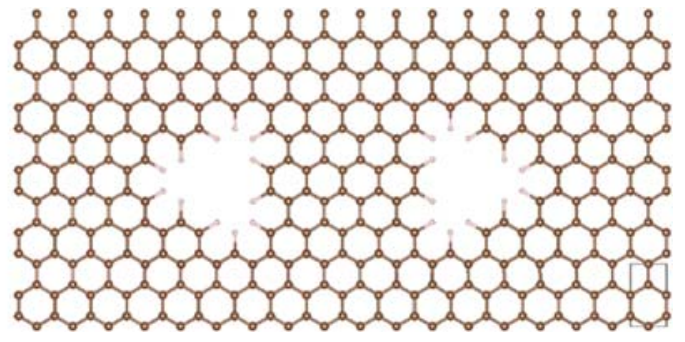

(a)

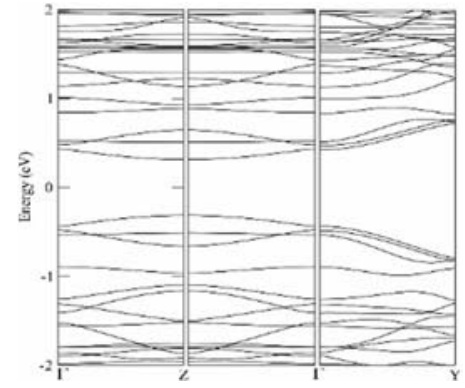

(b)

Figure 7. a) Placements of the holes; brown atoms denote $\mathrm{C}$ and pink atoms denote $\mathrm{H}$

b) bandstructure of the faceout structure 
Following the same procedure for the single hole structures, polarization change is calculated according to applied strain. As can be seen from the related figure, layouts of the triangular holes do not effect the change of the polarization so all of the structures gives the same piezoelectric coefficient $\sim 1.7 \times 10^{-10}$ $\mathrm{C} / \mathrm{m}^{2}$.

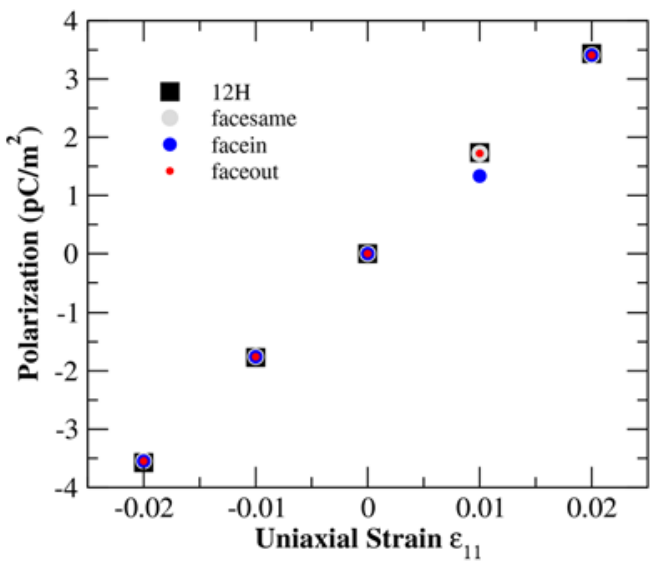

Figure 8. Polarization change under applied uniform strain for the facesame, facein and faceout structures

\section{DISCUSSION}

Experimentally observation of the formation of the triangular holes on 2D structures reinforces the reality of this study. Piezoelectricity is known as the natural property of the wurtzite structures. Today's widely used piezoelectric materials are lead based structures like lead zirconate titanate (PZT), but they are not environmental friendly substances because of the lead content. Researchers are in quest to find lead free piezoceramics like bismuth ferrite $\left(\mathrm{BiFeO}_{3}\right)$, bismuth titanate $\left(\mathrm{Bi}_{4} \mathrm{Ti}_{3} \mathrm{O}_{12}\right)$ but their efficiency and stabilities have not been confirmed yet. Also these materials are not eligible to use in nanodimensional devices. Successful experimental growth of the two dimensional materials enable researchers to negotiate this drawback. Electronical and optical properties of these 2D structures are widely studied, but it is still needed to investigate the piezoelectric properties of these materials. In the last few years, studies are focused on to solve this problem not only graphene but also transition metal dichalcogenides, II-IV group materials, $\mathrm{ZnO}$, etc. [8, 13, 30-36]. Experimental and theoretical calculations show that these monolayer materials are promising piezoelectric structures. Among them, graphene is one of the most known structure and needed to detailed investigation in view of piezoelectricity. Our results show that graphene can be used as piezoelectric material when coaxed with triangular holes. Size of the hole and the dimensions of the base graphene structure effects the piezoelectric coefficient values. If the results for the $12 \mathrm{H}$ and $18 \mathrm{H}$ structures are taken into account, inversely proportional ratio between the size of the holes and the piezoelectric coefficient. As the structures repeat themselves in all space because of the periodicity, same piezoelectric coefficient results for the $12 \mathrm{H}$ cell and the face-same structure can be predicted. Interestingly, layouts of the same sized triangular holes do not effect the piezoelectricity of the structure. Numerical results of this study shows that coaxed graphene is one of the good candidates for the 2D piezoelectric materials.

\section{RESULTS}

Naturally non-piezoelectric graphene can be shifted to piezoelectric material with nonsymmetric holes. Band gap values and the piezoelectricity can be tuned by changing the size of the hole. Piezoelectric coefficient of the structure is not effected by the layout of holes. Results of this study are comparable with conventional bulk piezoelectric materials. With the use of these $2 \mathrm{D}$ materials in low power electronic, sensors and nanoscale electromechanical systems are expected to have impact on size-weight reduction and energy consumption of such devices. 


\section{ACKNOWLEDGEMENTS}

Author acknowledges the support from Scientific and Technological Research Council of Turkey (TUBITAK-113F333). Also, computational resources were provided by TUBITAK ULAKBIM, High Performance and Grid Computing Center (TR-Grid e-Infrastructure).

\section{REFERENCES}

[1] Curie J, Curie P. Développement par compression de l'électricité polaire dans les cristaux hémièdres à faces inclinées(Development, via compression, of electric polarization in hemihedral crystals with inclined faces). Bulletin de la Société minérologique de France 1880; 3: 90 - 93.

[2] Song X, Hu J, Zeng H. Two-dimensional semiconductors: Recent progress and future perspectives. J Mater Chem C 2013; 1: 2952-2969.

[3] Xu M, Liang T, Shi M, Chen H. Graphene-like two-dimensional materials. Chem Rev 2013; 113 : 3766-3798.

[4] Lebègue S, Björkman T, Klintenberg M, Nieminen RM, Eriksson O. Two-dimensional materials from data filtering and ab initio calculations. Phys Rev X 2013; 3: 031002.

[5] Aliofkhazraaei M, Ali N, Milne WI, Ozkan CS, Mitura S, Gervasoni JL. Graphene science handbook: Size-dependent properties. New York, NY: Taylor \& Francis Group CRC Press, 2016.

[6] Zhu H, Wang Y, Xiao J, Liu M, Xiong S, Wong ZJ, Ye Z, Ye Y, Yin X, Zhang X. Observation of piezoelectricity in free-standing monolayer $\mathrm{MoS}_{2}$. Nat Nanotechnol 2014; 10: 151-155.

[7] Radisavljevic B, Radenovic A, Brivio J, Giacometti V, Kis A. Single-layer $\mathrm{MoS}_{2}$ transistors. Nat Nanotechnol 2011; 6: 147-150.

[8] Lee C, Yan H, Brus LE, Heinz TF, Hone J, Ryu S. Anomalous lattice vibrations of single- and fewlayer $\mathrm{MoS}_{2}$. ACS Nano 2010; 4: 2695-2700.

[9] Coleman JN, Lotya M, O’Neill A, Bergin SD, King PJ, Khan U, Young K, Gaucher A, De S, Smith RJ, et al. Two-dimensional nanosheets produced by liquid exfoliation of layered materials. Science 2011; 331: 568-571.

[10] Jin C, Lin F, Suenaga K, Lijima S. Fabrication of a freestanding boron nitride single layer and its defect assignments. Phys Rev Lett 2009; 102: 195505.

[11] Chandratre S, Sharma P. Coaxing graphene to be piezoelectric. Appl Phys Lett 2012; 100:023114.

[12] Ong MT, Reed EJ. Engineered piezoelectricity in graphene. ACS Nano 2012; 6: 1387-1394.

[13] Duerloo KAN, Ong MT, Reed EJ. Intrinsic piezoelectricity in two-dimensional materials. J Phys Chem Lett 2012; 3: 2871-2876.

[14] Chang Z, Yan W, Shang J, Liu JZ. Piezoelectric properties of graphene oxide: A first-principles computational study. Appl Phys Lett 2014; 105: 023103.

[15] Hangleiter A, Hitzel F, Lahmann S, Rossow U. Composition dependence of polarization fields in GaInN/GaN quantum wells. Appl Phys Lett 2003; 83: 1169. 
[16] Shimada K. First-principles determination of piezoelectric stress and strain constants of wurtzite III-V nitrides. Jpn J Appl Phys 2006; 45: L358-L360.

[17] Wang X, Tian H, Xie W, Shu Y, Mi WT, Mohammad MA, Xie QY, Yang Y, Xu JB, Ren TL. Observation of a giant two-dimensional band-piezoelectric effect on biaxial-strained graphene. NPG Asia Materials 2015; 7: e154.

[18] Rodrigues DC, Zelenovskiy P, Romanyuk K, Luchkin S, Kopelevich Y, Kholkin A. Strong piezoelectricity in single-layer graphene deposited on $\mathrm{SiO}_{2}$ grating substrates. Nat Comms 2015; 6: 7572.

[19] Morten B, Deccico G, Prudenziati M. Resonant pressure sensor based on piezoelectric properties of ferroelectric thick films. Sens Actuator A-Phys. 1992; 31: 153-158.

[20] Jaffe H, Berlincourt DA. Piezoelectric transducer materials. Proc IEEE 1965; 53:1372-1386.

[21] Wang ZL, Song JH. Piezoelectric nanogenerators based on zinc oxide nanowire arrays. Science 2006; 312: 242-245.

[22] Kresse G, Hafner J. Ab initio molecular dynamics for liquid metals. Phys Rev B 1993; 47: 558-561.

[23] Wu X, Vanderbilt D, Hamann DR. Systematic treatment of displacements, strains, and electric fields in density-functional perturbation theory. Phys Rev B 2005; 72: 035105-035117.

[24] Perdew JP, Burke K, Ernzerhof M. Generalized gradient approximation made simple. Phys Rev Lett 1996; 77: 3865-3868.

[25] Heyd J, Scuseria G, Ernzerhof M. Hybrid functionals based on a screened coulomb potential. J Chem Phys 2003; 118: 8207-8215.

[26] Fuchs F, Furthmueller J, Bechstedt F, Shishkin M, Kresse G. Quasiparticle band structure based on a generalized Kohn-Sham scheme. Phys Rev B: Condens Matter Mater Phys 2007; 76: 115109.

[27] Monkhorst HJ, Pack JD. Special points for Brillouin-zone integrations. Phys Rev B 1976; 13: 5188 5192.

[28] Vanderbilt D. Berry-phase theory of proper piezoelectric response. J Phys Chem Solids 2000; 61: $147-151$.

[29] Baroni S, de Gironcoli S, Dal Corso A, Giannozzi P. Phonons and related crystal properties from density-functional perturbation theory. Rev Mod Phys 2001; 73:515-562.

[30] Radisavljevic B, Radenovic A, Brivio J, Giacometti V, Kis A. Single-layer $\mathrm{MoS}_{2}$ transistors. Nat Nanotechnol 2011; 6: 147-150.

[31] Elias AL, Perea-Lopez N, Castro-Beltran A, Berkdemir A, Lv R, Feng S, Long AD, Takuya H, Kim YA, Endo M, et al. Controlled synthesis and transfer of large-area $\mathrm{WS}_{2}$ sheets: From single layer to few layers. ACS Nano 2013; 7: 5235-5242.

[32] Gutierrez HR, Perea-Lopez N, Elias AL, Berkdemir A, Wang B, Lv R, Lopez-Urias F, Crespi VH, Terrones $\mathrm{H}$, Terrones $\mathrm{M}$. Extraordinary room-temperature photoluminescence in triangular $\mathrm{WS}_{2}$ monolayers. Nano Lett 2013; 13: 3447-3454. 
Alyörük / Anadolu Univ. J. of Sci. and Technology A - Appl. Sci. and Eng. 17 (3) - 2016

[33] Huang JK, Pu J, Hsu CL, Chiu MH, Juang ZY, Chang YH, Chang WH, Iwasa Y, Takenobu T, Li LJ. Large-area synthesis of highly crystalline $\mathrm{WSe}_{2}$ monolayers and device applications. ACS Nano 2014; 8: 923-930.

[34] Liu W, Kang J, Sarkar D, Khatami Y, Jena D, Banerjee K. Role of metal contacts in designing high-performance monolayer n-type $\mathrm{WSe}_{2}$ field effect transistors. Nano Lett 2013; 13: 1983-1990.

[35] Mak KF, Lee C, Hone J, Shan J, Heinz TF. Atomically thin $\mathrm{MoS}_{2}$ : A new direct-gap semiconductor. Phys Rev Lett 2010; 105: 136805.

[36] Xiang HJ, Yang J, Hou JG, Zhu Q. Piezoelectricity in ZnO nanowires: A first-principles study. Appl Phys Lett 2006; 89: 223111-223114. 